\title{
GABOR FRAMES WITHOUT INEQUALITIES
}

\author{
KARLHEINZ GRÖCHENIG
}

\begin{abstract}
We prove fourteen equivalent conditions for a set of time-frequency shifts on a lattice $\Lambda,\left\{e^{2 \pi i \lambda_{2} \cdot t} g\left(t-\lambda_{1}\right):\left(\lambda_{1}, \lambda_{2}\right) \in \Lambda\right\} \subseteq L^{2}\left(\mathbb{R}^{d}\right)$, to be a frame for $L^{2}\left(\mathbb{R}^{d}\right)$. Remarkably, several of these conditions can be formulated without an inequality. In particular, instead of checking the invertibility of the frame operator on $L^{2}\left(\mathbb{R}^{d}\right)$, it suffices to verify that it is one-to-one on a certain subspace of tempered distributions.
\end{abstract}

\section{INTRODUCTION}

An operator on a finite-dimensional vector space is invertible, if and only if it is one-to-one. On an infinite-dimensional vector space, this characterization is hardly ever true and amounts to a mathematical miracle that says something deep about the operator.

In this paper we investigate the principle "injectivity implies invertibility" in the context of time-frequency analysis (phase-space analysis). We show that several operators associated to a Gabor frame are invertible on Hilbert space, if and only if they are one-to-one on a larger space. This insight seems to be completely new and is rather surprising.

Gabor frames are an important and convenient tool for phase-space expansions and the time-frequency analysis of distributions. The theory of Gabor frames is well developed, as is seen from the textbooks $[6,9,20]$. Several explicit constructions and many general characterizations are known. In all these characterizations the difficulty is to check the invertibility of some operator on a Hilbert space: this is either the associated frame operator on $L^{2}\left(\mathbb{R}^{d}\right)$, or some Gramian matrix on $\ell^{2}\left(\mathbb{Z}^{2 d}\right)$, or even of a whole family of matrices acting on $\ell^{2}\left(\mathbb{Z}^{d}\right)$. Equivalently, the invertibility amounts to finding a positive lower bound for an inequality, which is inevitably a hard problem.

Gabor frames are also implicit in Rieffel's work on projective modules over non-commutative tori [28] and thus play a (not yet fully understood) role in non-commutative geometry (see Luef's work on the connection between the two fields $[26])$.

We offer a number of new characterizations of Gabor frames that do not require inequalities or invertibility. In each case it suffices to verify the injectivity of some operators associated to a Gabor frame on a larger space instead proving

Date: March 13, 2007.

Key words and phrases. Gabor frame, modulation space, twisted convolution, injectivity implies invertibility.

K. G. was supported by the Marie-Curie Excellence Grant MEXT-CT 2004-517154. 
its invertibility on a Hilbert space. These results reveal a remarkable phenomenon in phase-space analysis and, to us, are completely unexpected. The results do not come easily: we will use some of the deepest results about Gabor frames, such as the duality theorem of Janssen and Ron-Shen, Wiener's Lemma for twisted convolution and the rotation algebra.

The paper is organized as follows. In Section 2 we collect the basic definitions required to formulate our results on Gabor frames. The main result is stated and commented in Section 3. It proof is carried out in Section 4 after a brief summary of several results from abstract harmonic analysis. Section 5 provides a few examples and further perspectives, such as the index of a Gabor system.

\section{Gabor Frames and Modulation Spaces - Basic Definitions}

To make our statements precise, let us introduce the main concepts required to treat Gabor frames. For the detailed exposition of Gabor frames and timefrequency analysis we refer to the books $[6,9,20]$.

Given a point $z=(x, \xi), x, \xi \in \mathbb{R}^{d}$, in phase space $\mathbb{R}^{2 d}$, we consider the phasespace shifts (time-frequency shifts) acting on a function $f$

$$
\pi(z) f(t)=M_{\xi} T_{x} f(t)=e^{2 \pi i \xi \cdot t} f(t-x), \quad t, x, \xi \in \mathbb{R}^{d} .
$$

A time-frequency lattice $\Lambda$ is a discrete subgroup of $\mathbb{R}^{2 d}$, of the form $\Lambda=A \mathbb{Z}^{2 d}$ for some invertible real-valued $2 d \times 2 d$-matrix. The adjoint lattice is $\Lambda^{\circ}=\left\{\mu \in \mathbb{R}^{2 d}\right.$ : $\pi(\lambda) \pi(\mu)=\pi(\mu) \pi(\lambda)$ for all $\lambda \in \Lambda\}$. If $\Lambda=\alpha \mathbb{Z}^{d} \times \beta \mathbb{Z}^{d}$, then $\Lambda^{\circ}=\beta^{-1} \mathbb{Z}^{d} \times \alpha^{-1} \mathbb{Z}^{d}$. The adjoint lattice should be distinguished from the dual lattice $\Lambda^{\perp}=\alpha^{-1} \mathbb{Z}^{d} \times$ $\beta^{-1} \mathbb{Z}^{d}$ that is more common is harmonic analysis.

Fix a $g \in L^{2}\left(\mathbb{R}^{d}\right)$, then the set of time-frequency shifts with respect to the lattice $\Lambda$ is denoted by

$$
\mathcal{G}(g, \Lambda)=\{\pi(\lambda) g: \lambda \in \Lambda\}
$$

and similarly $\mathcal{G}\left(g, \Lambda^{\circ}\right)$ is defined as $\mathcal{G}\left(g, \Lambda^{\circ}\right)=\left\{\pi(\mu) g: \mu \in \Lambda^{\circ}\right\}$. Sets of the form $\mathcal{G}(g, \Lambda)$ and $\mathcal{G}\left(g, \Lambda^{\circ}\right)$ are called Gabor systems.

Associated to every Gabor system there are four canonical operators: the analysis operator, the synthesis operator, the frame operator, and the Gramian. Precisely, these operators are defined as follows.

Definition 1. Fix a non-zero $g \in L^{2}\left(\mathbb{R}^{d}\right)$ and let $\Lambda \subseteq \mathbb{R}^{2 d}$ be a lattice. The coefficient operator $C_{g, \Lambda}$ associated to a test function $g$ and a lattice $\Lambda$ maps functions/distributions to sequences on $\Lambda$ and is defined to be

$$
\left(C_{g, \Lambda} f\right)(\lambda)=\langle f, \pi(\lambda) g\rangle \quad f \in L^{2}\left(\mathbb{R}^{d}\right), \lambda \in \Lambda .
$$

The synthesis operator $D_{g, \Lambda}$ maps sequences to functions/distributions and is

$$
D_{g, \Lambda} \mathbf{c}=\sum_{\lambda \in \Lambda} c_{\lambda} \pi(\lambda) g
$$

whenever the series is defined (for instance, for finite sequences $\mathbf{c}$ ).

The composition $S_{g, \Lambda}=D_{g, \Lambda} C_{g, \Lambda}$ is the frame operator corresponding to the Gabor system $\mathcal{G}(g, \Lambda)$ and maps functions to functions, when well-defined. 
Finally, the operator $G_{g, \Lambda}=C_{g, \Lambda} D_{g, \Lambda}$ is the Gramian operator mapping sequences indexed by $\Lambda$ to sequences. Viewed as a matrix, $G_{g, \Lambda}$ has the entries $G_{\lambda, \lambda^{\prime}}=\left\langle\pi\left(\lambda^{\prime}\right) g, \pi(\lambda) g\right\rangle$ for $\lambda, \lambda^{\prime} \in \Lambda$.

Definition 2. The set $\mathcal{G}(g, \Lambda)$ is called a Gabor frame (or Weyl-Heisenberg frame), if there exist constants $A, B>0$, such that

$$
A\|f\|_{2}^{2} \leq \sum_{\lambda \in \Lambda}|\langle f, \pi(\lambda) g\rangle|^{2} \leq B\|f\|_{2}^{2} \quad \text { for all } f \in L^{2}\left(\mathbb{R}^{d}\right) .
$$

Further, the set $\mathcal{G}(g, \Lambda)$ is called a Riesz sequence, if there exist constants $A^{\prime}, B^{\prime}>0$ such that

$$
A^{\prime}\|\mathbf{c}\|_{2} \leq\left\|\sum_{\lambda \in \Lambda} c_{\lambda} \pi(\lambda) g\right\|_{2} \leq B^{\prime}\|\mathbf{c}\|_{2}
$$

holds for all finite sequence $\mathbf{c}$.

Since $\langle S f, f\rangle=\sum_{\lambda \in \Lambda}|\langle f, \pi(\lambda) g\rangle|^{2}$, the inequalities in (5) express that the Gabor frame operator $S_{g, \Lambda}$ is bounded and invertible on $L^{2}\left(\mathbb{R}^{d}\right)$. To prove that $\mathcal{G}(g, \Lambda)$ is a frame, it is therefore necessary to either prove the invertibility of the operator $S_{g, \Lambda}$ or to prove the inequalities (5). Whereas the upper inequality is easy (it amounts to the boundedness of $S_{g, \Lambda}$ ), the lower lower inequality is difficult (it amounts to the invertibility of $\left.S_{g, \Lambda}\right)$. Several criteria for Gabor frames have been found, notably the Wexler-Raz conditions [10,25], the Ron-Shen duality [29], the characterization by the Ron-Shen matrices [20,29].

Likewise, $\mathcal{G}(g, \Lambda)$ is a Riesz sequence, if and only if the Gramian is invertible on $\ell^{2}\left(\mathbb{Z}^{d}\right)$. This follows from the identity

$$
\begin{aligned}
\left\|\sum_{\lambda \in \Lambda} c_{\lambda} \pi(\lambda) g\right\|_{2}^{2} & =\sum_{\lambda, \lambda^{\prime} \in \Lambda} c_{\lambda} \overline{c_{\lambda^{\prime}}}\left\langle\pi(\lambda) g, \pi\left(\lambda^{\prime}\right) g\right\rangle \\
& =\sum_{\lambda, \lambda^{\prime} \in \Lambda} c_{\lambda} \overline{\bar{c}_{\lambda^{\prime}}} G_{\lambda^{\prime}, \lambda}=\left\langle G_{g, \Lambda} \mathbf{c}, \mathbf{c}\right\rangle .
\end{aligned}
$$

We note that whenever a duality $\langle\cdot, \cdot\rangle$ is suitably defined and extends the inner product on $L^{2}\left(\mathbb{R}^{d}\right)$ (in particular, it is conjugate linear in the second term!), then the synthesis operator is adjoint to the analysis operator and vice versa, informally

$$
C_{g, \Lambda}^{*}=D_{g, \Lambda} .
$$

\section{Modulation Spaces.}

Definition 3. Fix a non-zero Schwartz function $\varphi$ (preferrably the Gaussian $\varphi(t)=$ $\left.e^{-\pi t \cdot t}\right)$. We say that a distribution $f \in \mathcal{S}^{\prime}\left(\mathbb{R}^{d}\right)$ belongs to the modulation space $M^{p}=$ $M^{p}\left(\mathbb{R}^{d}\right)$, if

$$
\|f\|_{M^{p}}:=\left(\int_{\mathbb{R}^{2 d}}|\langle f, \pi(z) \varphi\rangle|^{p} d z\right)^{1 / p}=\left\|V_{\varphi} f\right\|_{p}<\infty .
$$

Each $M^{p}\left(\mathbb{R}^{d}\right), 1 \leq p \leq \infty$, is a Banach space, and the definition is independent of the test function $\varphi \in \mathcal{S}\left(\mathbb{R}^{d}\right)$. If $1 \leq p<\infty$, then the dual space of $M^{p}$ is $M^{p^{\prime}}$ where $1 / p+1 / p^{\prime}=1[20$, Thm. 11.3.6]. We note that for $p=2$ we obtain 
$M^{2}=L^{2}\left(\mathbb{R}^{d}\right)$. See $[12-15,20]$ for the many beautiful properties of the modulation spaces and their many generalizations.

The transform

$$
\langle f, \pi(z) \varphi\rangle=\left\langle f, M_{\xi} T_{x} \varphi\right\rangle=\int_{\mathbb{R}^{d}} f(t) \overline{g(t-x)} e^{-2 \pi i x \cdot t} d t
$$

is the so-called short-time Fourier transform (also called Gabor transform, ambiguity function, coherent state transform). It measures the phase-space (timefrequency) content at $z=(x, \xi)$ in phase space. So $M^{1}$ consists of all those $L^{2}$ functions whose short-time Fourier transform is absolutely integrable, and $M^{\infty}$ contains exactly those tempered distributions with bounded short-time Fourier transform. It is not hard to see that the Schwartz class is a subspace of $M^{1}$. In time-frequency analysis and phase-space analysis, $M^{1}$ is therefore often used as an appropriate space of test functions, and $M^{\infty}$ serves as a suitable space of distributions $[12,19]$. The modulation spaces are tailored to the needs of time-frequency analysis, and they arise inevitably, whenever a problem involves the time-frequency shifts $\pi(z)$. For an exposition from scratch see [20, Ch. 11-13], for a detailed history and a comprehensive annotated list of references see Feichtinger's beautiful article [15].

With all definitions in place, we verify first when the operators associated to a Gabor system are bounded.

Lemma 2.1. Assume that $g \in M^{1}, g \neq 0$. Then

(i) $C_{g, \Lambda}$ maps $M^{p}\left(\mathbb{R}^{d}\right)$ into $\ell^{p}(\Lambda)$ and $\left\|C_{g, \Lambda} f\right\|_{p} \leq C\|g\|_{M^{1}}\|f\|_{M^{p}}$. The constant $C$ depends only on the lattice, but not on $g$ and $f$.

(ii) $D_{g, \Lambda}$ maps $\ell^{p}(\Lambda)$ into $M^{p}\left(\mathbb{R}^{d}\right)$ and $\left\|D_{g, \Lambda} \mathbf{c}\right\|_{p} \leq C\|g\|_{M^{1}}\|\mathbf{c}\|_{\ell^{p}}$.

(iii) The frame operator $S_{g, \Lambda}$ maps $M^{p}\left(\mathbb{R}^{d}\right)$ into $M^{p}\left(\mathbb{R}^{d}\right)$ and $\left\|S_{g, \Lambda} f\right\|_{p} \leq C^{2}\|g\|_{M^{1}}^{2}\|f\|_{M^{p}}$.

(iv) The Gramian $G_{g, \Lambda}$ maps $\ell^{p}(\Lambda)$ into $\ell^{p}(\Lambda)$ and $\left\|G_{g, \Lambda} \boldsymbol{c}\right\|_{p} \leq C^{2}\|g\|_{M^{1}}^{2}\|\boldsymbol{c}\|_{p}$.

These statements are well known and can be found in various sources, see [16] and [20, Cor. 12.1.12] for the proofs and detailed references.

For later use, we note that item (i) with $f=g$ implies that for $g \in M^{1}$ we have

$$
\sum_{\lambda \in \Lambda}|\langle g, \pi(\lambda) g\rangle|=\left\|C_{g, \Lambda} g\right\|_{1} \leq C\|g\|_{M_{1}}^{2}
$$

From (iii) and (iv) we see that the frame operator $S_{g, \Lambda}$ and the Gramian $G_{g, \Lambda}$ are always bounded. So the right-hand inequalities in (5) and (6) are always satisfied when $g \in M^{1}$.

To make sense of infinite series of time-frequency shifts, we need the following lemma.

Lemma 2.2. If $g \in M^{1}\left(\mathbb{R}^{d}\right)$ and $\mathbf{c} \in \ell^{\infty}(\Lambda)$, then the operator $\sum_{\lambda \in \Lambda} c_{\lambda} \pi(\lambda)$ is bounded from $M^{1}\left(\mathbb{R}^{d}\right)$ to $M^{\infty}\left(\mathbb{R}^{d}\right)$, and the sum converges unconditionally in the weak operator topology. 
Proof. By Lemma 2.1 we have

$$
\left\|C_{g, \Lambda} f\right\|_{1}=\sum_{\lambda \in \Lambda}|\langle f, \pi(\lambda) g\rangle| \leq C\|f\|_{M^{1}}\|g\|_{M^{1}} \quad \forall f, g \in M^{1} .
$$

Then we find that

$$
\begin{aligned}
\left|\left\langle\sum_{\lambda \in \Lambda} c_{\lambda} \pi(\lambda) f, g\right\rangle\right| & =\left|\sum_{\lambda \in \Lambda} c_{\lambda}\langle\pi(\lambda) f, g\rangle\right| \\
& \leq\|\mathbf{c}\|_{\infty} \sum_{\lambda \in \Lambda}|\langle\pi(\lambda) f, g\rangle| \leq C\|\mathbf{c}\|_{\infty}\|f\|_{M^{1}}\|g\|_{M^{1}} .
\end{aligned}
$$

Since $M^{1}$ and $M^{\infty}$ are dual to each other, this inequality implies that

$$
\left\|\sum_{\lambda \in \Lambda} c_{\lambda} \pi(\lambda) f\right\|_{M^{\infty}} \leq C\|f\|_{M^{1}} \quad \text { for all } f \in M^{1} .
$$

The weak unconditional convergence of $\sum_{\lambda} c_{\lambda} \pi(\lambda)$ follows immediately from (11).

Finally we need a strong form of linear independence of time-frequency shifts.

Proposition 2.3. If $\sum_{\lambda \in \Lambda} c_{\lambda} \pi(\lambda)=0$ for some $\boldsymbol{c} \in \ell^{\infty}(\Lambda)$, then $\boldsymbol{c}=0$.

Proof. By assumption we have, for all $g, h \in M^{1}$ and $z \in \mathbb{R}^{2 d}$,

$$
\sum_{\lambda \in \Lambda} c_{\lambda}\langle\pi(\lambda) \pi(z) g, \pi(z) h\rangle=0
$$

Now $\pi(z)^{-1} \pi(\lambda) \pi(z)=e^{2 \pi i[z, \lambda]} \pi(\lambda)$, where $[z, \lambda]=z_{1} \lambda_{2}-z_{2} \lambda_{1}$ is the symplectic form on $\mathbb{R}^{2 d}$. This implies that

$$
\sum_{\lambda \in \Lambda} c_{\lambda}\langle\pi(\lambda) g, h\rangle e^{2 \pi i \lambda_{2} z_{1}-\lambda_{1} z_{2}}=0
$$

for all $z \in \mathbb{R}^{2 d}$ and all $g, h \in M^{1}$.

Equation (12) is an absolutely converging Fourier series on $\mathbb{R}^{2 d} / \Lambda$. Since it vanishes everywhere, we must have

$$
c_{\lambda}\langle\pi(\lambda) g, h\rangle=0 \quad \forall \lambda \in \Lambda
$$

from which $c_{\lambda}=0$ for all $\lambda$.

\section{New Characterizations of Gabor Frames}

We are now ready to discuss the new criteria for Gabor frames. Precisely, for each of the operators associated to a Gabor system, we will state a property that is equivalent to the frame property. Conceptually, several of them are easier because they do not involve the invertibility or an inequality. The following theorem provides a characterization of a Gabor frame $\mathcal{G}(g, \Lambda)$ with a test function in $g \in M^{1}\left(\mathbb{R}^{d}\right)$ in terms of each of the associated operators $C, D$ and their combinations.

Theorem 3.1. Assume that $g \in M^{1}\left(\mathbb{R}^{d}\right), g \neq 0$. Then the following are equivalent:

(i) $\mathcal{G}(g, \Lambda)$ is a frame for $L^{2}\left(\mathbb{R}^{d}\right)$. 
(ii) $S_{g, \Lambda}$ is invertible on $M^{1}\left(\mathbb{R}^{d}\right)$.

(iii) $S_{g, \Lambda}$ is invertible on $M^{\infty}\left(\mathbb{R}^{d}\right)$.

(iv) $S_{g, \Lambda}$ is one-to-one on $M^{\infty}\left(\mathbb{R}^{d}\right)$.

(v) $C_{g, \Lambda}$ is one-to-one from $M^{\infty}\left(\mathbb{R}^{d}\right)$ to $\ell^{\infty}(\Lambda)$.

(vi) $D_{g, \Lambda}$ defined on $\ell^{1}(\Lambda)$ has dense range in $M^{1}\left(\mathbb{R}^{d}\right)$.

(vii) $D_{g, \Lambda}$ is surjective from $\ell^{1}(\Lambda)$ onto $M^{1}\left(\mathbb{R}^{d}\right)$.

(viii) $D_{g, \Lambda^{\circ}}$ is one-to-one from $\ell^{\infty}\left(\Lambda^{\circ}\right)$ to $M^{\infty}\left(\mathbb{R}^{d}\right)$.

(ix) $C_{g, \Lambda^{\circ}}$ defined on $M^{1}\left(\mathbb{R}^{d}\right)$ has dense range in $\ell^{1}(\Lambda)$.

(x) $C_{g, \Lambda^{\circ}}$ is surjective from $M^{1}\left(\mathbb{R}^{d}\right)$ onto $\ell^{1}(\Lambda)$.

(xi) $G_{g, \Lambda^{\circ}}$ is invertible on $\ell^{1}\left(\Lambda^{\circ}\right)$.

(xii) $G_{g, \Lambda^{\circ}}$ is invertible on $\ell^{\infty}\left(\Lambda^{\circ}\right)$.

(xiii) $G_{g, \Lambda^{\circ}}$ is one-to-one on $\ell^{\infty}\left(\Lambda^{\circ}\right)$.

(xiv) $\mathcal{G}\left(g, \Lambda^{\circ}\right)$ is a Riesz sequence in $L^{2}\left(\mathbb{R}^{d}\right)$.

The merit of Theorem 3.1 is its beauty and completeness. Conceptually it is simpler to verify the injectivity of an operator than to prove its invertibility or its surjectivity. To emphasize this point, let us single out two conditions and state separately the following simplified version of Theorem 3.1. The following was the original conjecture from which Theorem 3.1 evolved.

Corollary 3.2. Assume that $g \in M^{1}\left(\mathbb{R}^{d}\right)$ and $\Lambda$ is a lattice in $\mathbb{R}^{2 d}$ with adjoint lattice $\Lambda^{\circ}$. Then the following are equivalent:

(i) The set $\mathcal{G}(g, \Lambda)$ is a frame for $L^{2}\left(\mathbb{R}^{d}\right)$.

(ii) The analysis operator $C_{g, \Lambda}$ is one-to-one from $M^{\infty}\left(\mathbb{R}^{d}\right)$ to $\ell^{\infty}(\Lambda)$.

(iii) The synthesis operator $D_{g, \Lambda^{\circ}}$ is one-to-one from $\ell^{\infty}(\Lambda)$ to $M^{\infty}\left(\mathbb{R}^{d}\right)$.

The verification of conditions (ii) or (iii) may be easier in some cases. Each of these yields the frame property of $\mathcal{G}(g, \Lambda)$, without involving any inequality or the inversion of an operator. This insight seems to be completely new and is rather surprising. In Section 5 we will show how Corollary 3.2 can be used for counterexamples.

\section{Diskussion of Theorem 3.1.}

1. The set of conditions (viii) - (xiv) is dual to the set of conditions (i) - (vii). The dual conditions are obtained by replacing the lattice $\Lambda$ by its adjoint lattice $\Lambda^{\circ}$ and interchanging the indices 1 and $\infty$ or interchanging the role of $C$ and $D$.

The equivalence of (i) and (xiv) is the so-called Ron-Shen duality for Gabor frames. It is implicit in the work of Rieffel [28], was first obtained by Janssen [25] and then independently by Ron and Shen [29] and Daubechies, Landau, and Landau [10]. The version for arbitrary lattices is due to Feichtinger and Kozek [18]. This duality is a fundamental principle in the time-frequency analysis of Gabor frames and is connected with deep results in operator theory [28]. Theorem 3.1 extends the duality theory of Gabor frames.

2. It is well known that Gabor frames with windows in $M^{1}$ extend to so-called Banach frames for the modulation spaces. One way to formulate this fact is the following: For $g \in M^{1}$ the following properties are equivalent. (a) The frame 
operator $S_{g, \Lambda}$ is invertible on $L^{2}$. (b) $S_{g, \Lambda}$ is invertible on $M^{p}$ for some $p, 1 \leq p \leq$ $\infty$. (c) $S_{g, \Lambda}$ is simultaneously invertible on all $M^{p}, 1 \leq p \leq \infty$. See [16, Thm. 5.2] and [20, Prop. 12.2.7] and the theory of Gelfand triples [18]. Whereas this theorem suggests that all modulation spaces play the same role, Theorem 3.1 shows that the situation in not entirely symmetric and that $M^{\infty}$ plays a distinguished role. On $M^{\infty}$ the frame operator $S_{g, \Lambda}$ is invertible, if and only if it is one-to-one. This is far from true on $L^{2}=M^{2}$. It is well-known that $S_{g, \Lambda}$ may be one-to-one on $L^{2}$ without being invertible. See the examples in Section 5.

\section{Proof of Theorem 3.1}

Before we begin with the proof, we collect some facts from abstract harmonic analysis. In particular, we review Wiener's Lemma for twisted convolution and its role in the analysis of Gabor frames.

Some of the implications of Theorem 3.1 are quite easy to prove, but others require the full arsenal of time-frequency analysis. We will apply some of the deepest results in time-frequency analysis. We only give a short explanation of the results needed and place them in the context in time-frequency analysis. Full details are found in the cited literature.

4.1. Twisted Convolution. Recall first time-frequency shifts do not commute. If $\lambda=\left(\lambda_{1}, \lambda_{2}\right) \in \mathbb{R}^{d} \times \mathbb{R}^{d} \simeq \mathbb{R}^{2 d}$ and $\mu=\left(\mu_{1}, \mu_{2}\right) \in \mathbb{R}^{2 d}$, then their composition is

$$
\pi(\lambda) \pi(\mu)=e^{-2 \pi i \lambda_{1} \cdot \mu_{2}} \pi(\lambda+\mu) .
$$

The occurring phase factor defines a quadratic form $\sigma$ on $\mathbb{R}^{2 d}$ by

$$
\sigma(\lambda, \mu)=\lambda_{1} \cdot \mu_{2}
$$

Let $\mathbf{a}, \mathbf{b}$ be two finite sequences indexed by the lattice $\Lambda \subseteq \mathbb{R}^{2 d}$. The twisted convolution $\mathbf{a} \downarrow \mathbf{b}$ is defined to be

$$
(\mathbf{a} \bigsqcup \mathbf{b})(\lambda)=\sum_{\mu \in \Lambda} a_{\lambda} b_{\mu-\lambda} e^{2 \pi i \sigma(\lambda, \mu-\lambda)}
$$

Strictly speaking, the twisted convolution depends on the lattice $\Lambda$ and we would have to write $\natural_{\Lambda}$. However, since we use only a fixed lattice $\Lambda$ and its adjoint lattice $\Lambda^{\circ}$, no confusion can arise, and we will omit the subscript.

By Young's inequality the twisted convolution extends to certain $\ell^{p}$-spaces; in particular we have $\|\mathbf{a} \natural \mathbf{b}\|_{p} \leq\|\mathbf{a}\|_{p}\|\mathbf{b}\|_{1}$ for $\mathbf{a} \in \ell^{p}(\Lambda)$ and $\mathbf{b} \in \ell^{1}(\Lambda)$.

In our context the fundamental property of twisted convolution is Wiener's Lemma for twisted convolution.

Theorem 4.1. Assume that $\boldsymbol{a} \in \ell^{1}(\Lambda)$ and that the (twisted convolution) operator $C_{\boldsymbol{a}} \boldsymbol{c}=\boldsymbol{c} \llbracket \boldsymbol{a}$ is invertible on $\ell^{2}(\Lambda)$. Then the inverse is $C_{\boldsymbol{a}}^{-1}=C_{\boldsymbol{b}}$ for the unique $\boldsymbol{b} \in \ell^{1}(\Lambda)$. Consequently, $C_{\boldsymbol{a}}$ is invertible simultaneously on all $\ell^{p}(\Lambda), 1 \leq p \leq \infty$.

For the proof and more general statements see [22], for alternative proofs see [3, $23]$. 
4.2. Twisted Convolution in Time-Frequency Analysis. Twisted convolution arises in several contexts in time-frequency analysis, sometimes naturally, sometimes hidden.

(A) Consider series of time-frequency shifts of the form $\pi(\mathbf{c})=\sum_{\lambda \in \Lambda} c_{\lambda} \pi(\lambda)$. If $\mathbf{c} \in \ell^{\infty}(\Lambda)$, then by Lemma $2.2 \pi(\mathbf{c})$ is bounded from the space of test functions $M^{1}$ to the space of distributions $M^{\infty}$, so $\pi(\mathbf{c})$ is always well defined. If $\mathbf{c} \in \ell^{1}(\Lambda)$, then $\pi(\mathbf{c})$ is an absolutely convergent series of time-frequency shifts and is easily seen to be bounded on each $M^{p}, 1 \leq p \leq \infty$.

Let $\mathbf{a}, \mathbf{b} \in \ell^{1}(\Lambda)$, then the commutation rule (13) implies that

$$
\pi(\mathbf{a}) \pi(\mathbf{b})=\pi(\mathbf{a} \bigsqcup \mathbf{b})
$$

Furthermore, $\pi: \ell^{1}(\Lambda) \rightarrow \mathcal{B}\left(L^{2}\left(\mathbb{R}^{d}\right)\right)$ is a faithful representation of the (involutive) Banach algebra $\ell^{1}(\Lambda)$ with respect to $\emptyset$ into the bounded operators on $L^{2}\left(\mathbb{R}^{d}\right)([22]$ or [28]). With some work, Wiener's Lemma for twisted convolution can be transferred to the operator algebra $\pi\left(\ell^{1}(\Lambda)\right)$ of absolutely convergent time-frequency shifts, see [22]. As a result we state Wiener's Lemma for the so-called rotation algebra $\pi\left(\ell^{1}(\Lambda)\right)$.

Theorem 4.2. Let $S=\sum_{\lambda \in \Lambda} a_{\lambda} \pi(\lambda)=\pi(\boldsymbol{a})$. If $\boldsymbol{a} \in \ell^{1}(\Lambda)$ and $S$ is invertible on $L^{2}\left(\mathbb{R}^{d}\right)$, then $S^{-1}$ is again an absolutely convergent series of time-frequency shifts $S^{-1}=\sum_{\lambda \in \Lambda} b_{\lambda} \pi(\lambda)=\pi(\boldsymbol{b})$ for a unique $\boldsymbol{b} \in \ell^{1}(\Lambda)$ satisfying $\boldsymbol{a} \bigsqcup \boldsymbol{b}=\boldsymbol{b} \llbracket \boldsymbol{a}=\delta$. As a consequence, $S$ is invertible simultaneously on all modulation spaces $M^{p}, 1 \leq p \leq$ $\infty$.

REMARK: Although we do not need it here, we would like to point out an interesting and deep relation between Gabor frames and operator algebras and noncommutative geometry discovered by Luef [26]. In the language of operator algebras, $\pi\left(\ell^{1}(\Lambda)\right)$ is a rotation algebra or non-commutative torus, and Wiener's Lemma is usually referred to as the spectral invariance property or the spectral permanence $[1,8]$. If the coefficient algebra $\ell^{1}(\Lambda)$ is replaced by the Frechet algebra $\mathcal{S}(\Lambda)$ of rapidly decaying sequences, the corresponding version of Theorem 4.2 is a celebrated theorem of Connes about the spectral invariance of smooth noncommutative tori [7].

(B) The Gramian Operator. Recall that

$$
G_{\lambda, \mu}=\langle\pi(\mu) g, \pi(\lambda) g\rangle=e^{2 \pi i \sigma(\mu, \lambda-\mu)}\langle g, \pi(\lambda-\mu) g\rangle .
$$

Writing $a_{\lambda}=\langle g, \pi(\lambda) g\rangle$, the action of the Gramian operator $G$ can be written as a twisted convolution:

$$
\begin{aligned}
(G \mathbf{c})_{\lambda} & =\sum_{\mu \in \Lambda} G_{\lambda \mu} c_{\mu} \\
& =\sum_{\mu \in \Lambda} c_{\mu}\langle g, \pi(\lambda-\mu) g\rangle e^{2 \pi i \sigma(\mu, \lambda-\mu)} \\
& =(\mathbf{c} \downarrow \mathbf{a})_{\lambda} .
\end{aligned}
$$


This identity makes is plausible why Theorem 4.1 enters the proof of our main result.

(C) Janssen's representation of the Gabor frame operator. The frame operator $S_{g, \Lambda}$ commutes with all time-frequency shifts $\pi(\lambda), \lambda \in \Lambda$, and thus belongs to the commutants of $\pi(\Lambda)$. By definition of the adjoint lattice, this commutant is spanned by the time-frequency shifts $\left\{\pi(\mu), \mu \in \Lambda^{\circ}\right\}$. It is therefore plausible that $S_{g, \Lambda}$ can be represented by a sum of time-frequency shifts over the adjoint lattice $\Lambda^{\circ}$ in some sense. The precise statement is Janssen's representation [25] of the frame operator.

Theorem 4.3. If $g \in M^{1}$, then $S_{g, \Lambda}=\sum_{\mu \in \Lambda^{\circ}} a_{\mu} \pi(\mu)=\pi(\boldsymbol{a})$ for some $\boldsymbol{a} \in \ell^{1}\left(\Lambda^{\circ}\right)$. The coefficients are given explicitly by $a_{\mu}=s(\Lambda)^{-1}\langle g, \pi(\mu) g\rangle$, where $s(\Lambda)=|\operatorname{det} A|$ is the size of $\Lambda=A \mathbb{Z}^{2 d}$.

If, in addition, $\mathcal{G}(g, \Lambda)$ is a frame, then $S_{g, \Lambda}$ is invertible and Theorem 4.2 implies that $S_{g, \Lambda}$ is invertible simultaneously on all modulation spaces $M^{p}, 1 \leq p \leq \infty$.

4.3. Plan of the Proof. We will prove the following chain of implications. First we cover all conditions involving the lattice $\Lambda$ and show how they imply condition (viii) involving the adjoint lattice $\Lambda^{\circ}$. We will prove the implications

$$
(i) \Rightarrow(i i) \Rightarrow(i i i) \Rightarrow(i v) \Rightarrow(v) \Rightarrow(v i) \Rightarrow(v i i i)
$$

and

$$
(\text { ii) } \Rightarrow(v i i) \Rightarrow(v i i i)
$$

On the side of the adjoint lattice $\Lambda^{\circ}$ we will prove the following implications:

$$
(\text { viii }) \Rightarrow(i x) \Rightarrow(x) \Rightarrow(x i v) \Rightarrow(i)
$$

and

$$
(i) \Rightarrow(x i v) \Rightarrow(x i) \Rightarrow(x i i) \Rightarrow(x i i i) \Rightarrow(v i i i)
$$

Let us start!

4.4. Proof. (i) $\Leftrightarrow$ (ii). The simultaneous invertibility of the frame operator $S_{g, \Lambda}$ on $L^{2}\left(\mathbb{R}^{d}\right), M^{1}\left(\mathbb{R}^{d}\right)$, and $M^{\infty}\left(\mathbb{R}^{d}\right)$ is the main theorem of [22]; For the special lattices $\Lambda=\alpha \mathbb{Z}^{d} \times \beta \mathbb{Z}^{d}$ with rational $\alpha \beta$ this fact was already proved in $[16$, Thm. 5.2]. See Section 4.2 for a discussion of the context.

(ii) $\Leftrightarrow$ (iii). The frame operator $S_{g, \Lambda}$ is self-adjoint. Hence it is invertible on $M^{1}\left(\mathbb{R}^{d}\right)$ if and only if it is invertible on the dual space $M^{\infty}\left(\mathbb{R}^{d}\right)$.

(iii) $\Rightarrow$ (iv) is obvious.

(iv) $\Rightarrow(\mathbf{v})$. If $S_{g, \Lambda}=D_{g, \Lambda} C_{g, \Lambda}$ is one-to-one on $M^{\infty}\left(\mathbb{R}^{d}\right)$, then clearly $C_{g, \Lambda}$ must be one-to-one from $M^{\infty}\left(\mathbb{R}^{d}\right)$ to $\ell^{\infty}(\Lambda)$.

$(\mathbf{v}) \Leftrightarrow(\mathbf{v i})$. The adjoint operator of $D_{g, \Lambda}: \ell^{1}(\Lambda) \rightarrow M^{1}\left(\mathbb{R}^{d}\right)$ is exactly $C_{g, \Lambda}: M^{\infty}\left(\mathbb{R}^{d}\right) \rightarrow \ell^{\infty}(\Lambda)$. So $C_{g, \Lambda}$ is one-to-one, if and only if its adjoint $D_{g, \Lambda}$ has dense range. 
(vi), (vii) $\Rightarrow$ (viii). By assumption $D_{g, \Lambda}\left(\ell^{1}(\Lambda)\right)$ is a dense subspace of $M^{1}\left(\mathbb{R}^{d}\right)$ (or equals $M^{1}\left(\mathbb{R}^{d}\right)$ ). Then by Lemma 2.1(ii) the finite linear combinations of the form $f=\sum_{\lambda} a_{\lambda} \pi(\lambda) g \in M^{1}\left(\mathbb{R}^{d}\right)$ span also a dense subspace of $M^{1}$. Now assume that

$$
\sum_{\mu \in \Lambda^{\circ}} c_{\mu} \pi(\mu) g=0
$$

for some $\mathbf{c} \in \ell^{\infty}\left(\Lambda^{\circ}\right)$ (as a distribution in $\left.M^{\infty}\right)$. Let $f=\sum_{\lambda \in F} a_{\lambda} \pi(\lambda) g \in M^{1}\left(\mathbb{R}^{d}\right)$ for some finite set $F \subseteq \Lambda$. Since $\pi(\lambda), \lambda \in \Lambda$ and $\pi(\mu), \mu \in \Lambda^{\circ}$ commute, we find that

$$
\sum_{\mu \in \Lambda^{\circ}} c_{\mu} \pi(\mu) f=\sum_{\mu \in \Lambda^{\circ}} c_{\mu} \pi(\mu)\left(\sum_{\lambda \in F} a_{\lambda} \pi(\lambda) g\right)=\sum_{\lambda \in F} a_{\lambda} \pi(\lambda)\left(\sum_{\mu \in \Lambda^{\circ}} c_{\mu} \pi(\mu) g\right)=0 .
$$

This calculation is justified by Lemma 2.2. Thus $\sum_{\mu} c_{\mu} \pi(\mu): M^{1} \rightarrow M^{\infty}$ vanishes on a dense subspace of $M^{1}$, consequently, $\sum_{\mu} c_{\mu} \pi(\mu)=0$. Since time-frequency shifts are linearly independent by Proposition 2.3, it follows that $\mathbf{c}=0$.

(ii) $\Rightarrow$ (vii). If $S_{g, \Lambda}=D_{g, \Lambda} C_{g, \Lambda}$ is a bijection on $M^{1}$, then $D_{g, \Lambda}$ must be surjective from $\ell^{1}(\Lambda)$ onto $M^{1}\left(\mathbb{R}^{d}\right)$.

(viii) $\Leftrightarrow$ (ix). Again, $C_{g, \Lambda^{\circ}}: M^{1} \rightarrow \ell^{1}\left(\Lambda^{\circ}\right)$ has dense range, if and only if its adjoint operator $D_{g, \Lambda^{\circ}}: \ell^{\infty}\left(\Lambda^{\circ}\right) \rightarrow M^{\infty}$ is one-to-one.

(ix) $\Rightarrow(\mathrm{x})$. Assume that the analysis operator $C_{g, \Lambda^{\circ}}$ from $M^{1}$ has dense range in $\ell^{1}\left(\Lambda^{\circ}\right)$. Then for any fixed $\epsilon, 0 \leq \epsilon<1$, there is a function $\varphi \in M^{1}\left(\mathbb{R}^{d}\right)$ such that $\left\|C_{g, \Lambda^{\circ}} \varphi-\delta\right\|_{1}<\epsilon$, explicitly,

$$
\sum_{\mu \in \Lambda^{\circ}}\left|\langle\varphi, \pi(\mu) g\rangle-\delta_{\mu, 0}\right|=|\langle\varphi, g\rangle-1|+\sum_{\mu \in \Lambda^{\circ}, \mu \neq 0} \mid\langle\varphi, \pi(\mu) g|<\epsilon .
$$

Now let $\Phi$ be the matrix defined by the entries

$$
\Phi_{\mu \nu}=\langle\pi(\nu) \varphi, \pi(\mu) g\rangle \quad \mu, \nu \in \Lambda^{\circ} .
$$

( $\Phi$ is the "cross Gramian" of the Gabor systems $\mathcal{G}\left(g, \Lambda^{\circ}\right)$ and $\mathcal{G}\left(\varphi, \Lambda^{\circ}\right)$.) We will show that $\Phi$ is invertible by applying Schur's test to $\Phi-$ I. First we estimate the operator norm on $\ell^{1}$ :

$$
\begin{aligned}
\|\Phi-\mathrm{I}\|_{\ell^{1} \rightarrow \ell^{1}} & =\sup _{\nu \in \Lambda^{\circ}} \sum_{\mu \in \Lambda^{\circ}}\left|\Phi_{\mu \nu}-\delta_{\mu \nu}\right| \\
& =\sup _{\nu \in \Lambda^{\circ}} \sum_{\mu \in \Lambda^{\circ}}\left|\langle\pi(\nu) \varphi, \pi(\mu) g\rangle-\delta_{\mu \nu}\right| \\
& =\sup _{\nu \in \Lambda^{\circ}}\left(|\langle\varphi, g\rangle-1|+\sum_{\mu \in \Lambda^{\circ}, \mu \neq \nu}|\langle\varphi, \pi(\mu-\nu) g\rangle|\right) \\
& =|\langle\varphi, g\rangle-1|+\sum_{\mu \in \Lambda^{\circ}, \mu \neq 0}|\langle\varphi, \pi(\mu) g\rangle| \\
& =\left\|C_{g, \Lambda^{\circ}} \varphi-\delta\right\|_{1}<\epsilon .
\end{aligned}
$$

Since $\|\Phi-\mathrm{I}\|_{\ell^{1} \rightarrow \ell^{1}}<\epsilon<1, \Phi$ is invertible on $\ell^{1}\left(\Lambda^{\circ}\right)$. 
Now let $\mathbf{a} \in \ell^{1}\left(\Lambda^{\circ}\right)$ be arbitrary, then there exists $\mathbf{c} \in \ell^{1}\left(\Lambda^{\circ}\right)$, such that $\Phi \mathbf{c}=\mathbf{a}$. Set $f=\sum_{\nu \in \Lambda^{\circ}} c_{\nu} \pi(\nu) \varphi$, then

$$
\begin{aligned}
\left(C_{g, \Lambda^{\circ}} f\right)(\mu) & =\langle f, \pi(\mu) g\rangle \\
& =\sum_{\nu \in \Lambda^{\circ}} c_{\nu}\langle\pi(\nu) \varphi, \pi(\mu) g\rangle \\
& =(\Phi \mathbf{c})(\mu)=a_{\mu} .
\end{aligned}
$$

Thus $C_{g, \Lambda^{\circ}}$ is surjective from $M^{1}$ onto $\ell^{1}\left(\Lambda^{\circ}\right)$.

(x) $\Rightarrow\left(\right.$ xiv). If $C_{g, \Lambda^{\circ}}$ is surjective from $M^{1}$ onto $\ell^{1}\left(\Lambda^{\circ}\right)$, then there exists a function $\gamma \in M^{1}\left(\mathbb{R}^{d}\right)$, a so-called dual window, such that

$$
\langle\gamma, \pi(\mu) g\rangle=\delta_{\mu, 0} \quad \text { for all } \mu \in \Lambda^{\circ} .
$$

(This is the so-called Wexler-Raz biorthogonality condition and known to be equivalent to $\mathcal{G}(g, \Lambda)$ being a frame $[10,25]$. So condition (x) implies (i) directly.) Consequently, we also have

$$
\langle\pi(\nu) \gamma, \pi(\mu) g\rangle=\delta_{\mu, \nu} \quad \text { for all } \mu, \nu \in \Lambda^{\circ} .
$$

If $f=\sum_{\mu \in \Lambda^{\circ}} c_{\mu} \pi(\mu) g=D_{g, \Lambda^{\circ}} \mathbf{c}$, then, according to (25), the coefficients are determined by

$$
\langle f, \pi(\nu) \gamma\rangle=\sum_{\mu \in \Lambda^{\circ}} c_{\mu}\langle\pi(\mu) g, \pi(\nu) \gamma\rangle=\sum_{\mu \in \Lambda^{\circ}} c_{\mu} \delta_{\mu \nu}=c_{\nu}
$$

In different notation,

$$
\mathbf{c}=C_{\gamma, \Lambda^{\circ}} f,
$$

and by the boundedness of $C_{\gamma, \Lambda^{\circ}}$ (Lemma 2.1(i)) we find that

$$
\|\mathbf{c}\|_{2}=\left\|C_{\gamma, \Lambda^{\circ}} f\right\|_{2} \leq C\|\gamma\|_{M^{1}}\|f\|_{2}=C\|\gamma\|_{M_{1}}\left\|D_{\gamma, \Lambda^{\circ}} \mathbf{c}\right\|_{2} \leq C^{2}\|\gamma\|_{M^{1}}^{2}\|\mathbf{c}\|_{2}
$$

for all $\mathbf{c} \in \ell^{2}\left(\Lambda^{\circ}\right)$. This inequality says that $\mathcal{G}\left(g, \Lambda^{\circ}\right)$ is a Riesz sequence, and we have proved (xiv).

(xiv) $\Leftrightarrow$ (i). This equivalence is known as the duality principle for Gabor frames and well known. See the discussion in Section 3 and $[25,29]$.

(xiv) $\Rightarrow(\mathbf{x i})$, (xii). We have already seen in (16) that the action of the Gramian operator can be recast as a twisted convolution operator. Set $a_{\mu}=$ $\langle g, \pi(\mu) g\rangle$ for $\mu \in \Lambda^{\circ}$, then $\mathbf{a} \in \ell^{1}\left(\Lambda^{\circ}\right)$ by (9) and (16) expresses the action of $G$ as a twisted convolution:

$$
G \mathbf{c}=\mathbf{c} \downarrow \mathbf{a} .
$$

Now assume that $\mathcal{G}\left(g, \Lambda^{\circ}\right)$ is a Riesz sequence, then the Gramian operator $G$ (and thus the twisted convolution operator) is invertible on $\ell^{2}\left(\Lambda^{\circ}\right)$. By Wiener's Lemma (Theorem 4.1) $G^{-1}$ is again a twisted convolution operator $G^{-1} \mathbf{c}=\mathbf{c} \natural \mathbf{b}$ for some $\mathbf{b} \in \ell^{1}\left(\Lambda^{\circ}\right)$. Consequently $G^{-1}$ is invertible on all $\ell^{p}\left(\Lambda^{\circ}\right), 1 \leq p \leq \infty$, as was to be proved.

(xi) $\Leftrightarrow$ (xii). The matrix $G_{g, \Lambda^{\circ}}$ is self-adjoint. Hence it is invertible on $\ell^{1}\left(\Lambda^{\circ}\right)$ if and only if if it is invertible on the dual space $\ell^{\infty}\left(\Lambda^{\circ}\right)$.

(xii) $\Rightarrow$ (xiii) is obvious. 
(xiii) $\Rightarrow$ (viii). If $G_{g, \Lambda^{\circ}}=C_{g, \Lambda^{\circ}} D_{g, \Lambda^{\circ}}$ is one-to-one on $\ell^{\infty}\left(\Lambda^{\circ}\right)$, then clearly $D_{g, \Lambda^{\circ}}$ must be one-to-one from $\ell^{\infty}\left(\Lambda^{\circ}\right)$ to $M^{\infty}\left(\mathbb{R}^{d}\right)$.

We have now shown that all fourteen conditions are equivalent and the proof is complete.

\section{Examples and Further Topics}

We conclude with several examples and some variations.

In general, Theorem 3.1 will not make it easier to verify that a particular Gabor system $\mathcal{G}(g, \Lambda)$ is a frame. However, Theorem 3.1 can be used to show that $\mathcal{G}(g, \Lambda)$ fails to be a frame by constructing explicit sequences in the kernel of $D_{g, \Lambda^{\circ}}$. We will do this in two (known) cases. The benefit of Theorem 3.1 is again that no inequalities have to be proved or disproved.

(a) Let $g(t)=e^{-\pi t^{2}}$ be the Gaussian and $\Lambda=\Lambda^{\circ}=\mathbb{Z}^{2}$. It was shown in [4] that the synthesis operator $D_{g, \mathbb{Z}^{2}}$ is one-to-one on $\ell^{2}\left(\mathbb{Z}^{2}\right)$ and hence the linear combinations of time-frequency shifts of the Gaussian are dense in $L^{2}(\mathbb{R})$. This fact was already claimed by J. von Neumann [27], see also $[2,24]$. By contrast, $D_{g, \mathbb{Z}^{2}}$ is not one-to-one on $\ell^{\infty}\left(\mathbb{Z}^{2}\right)$. By Theorem 3.1 the Gabor system $\mathcal{G}\left(g, \mathbb{Z}^{2}\right)$ cannot be a frame for $L^{2}(\mathbb{R})$. An example in the kernel of $D_{g, \mathbb{Z}^{2}}$ is the sequence $c_{k l}=(-1)^{k+l}$. One verifies that

$$
\sum_{k, l \in \mathbb{Z}}(-1)^{k+l} e^{2 \pi i k t} e^{-\pi(t-l)^{2}}=0
$$

as a distribution in $M^{\infty}(\mathbb{R})$. In fact, $\operatorname{ker} D_{g, \mathbb{Z}^{2}}=\mathbb{C} \mathbf{c}$, the kernel has dimension 1. Consequently, the synthesis operator $D_{g, \mathbb{Z}^{2}}$ is also one-to-one on $\ell^{p}$ for $p<\infty$. This example shows that one may not hope for a better result in Theorem 3.1.

(b) If $g \in M^{1}(\mathbb{R})$ satisfies the partition-of-unity condition

$$
\sum_{k \in \mathbb{Z}^{d}} g(t-\gamma k)=a \neq 0 \quad \text { for all } t \in \mathbb{R}^{d},
$$

then the Gabor system $\mathcal{G}(g, \Lambda)$ cannot be a frame for any lattice of the form $\Lambda=$ $\alpha \mathbb{Z}^{d} \times \frac{N}{\gamma} \mathbb{Z}^{d}$, where $\alpha>0$ is arbitrary and $N=2,3,4, \ldots$ This was proved in $[11,21]$. Theorem 3.1 offers a simple proof of this fact: we have to show that $D_{g, \Lambda^{\circ}}$ possesses a non-trivial kernel in $\ell^{\infty}$. We first write

$$
D_{g, \Lambda^{\circ}} \mathbf{c}=\sum_{k, l \in \mathbb{Z}} c_{k l} M_{\ell / \alpha} T_{k / \beta} g=\sum_{k \in \mathbb{Z}} m_{k} T_{k / \beta} g
$$

for a sequence of $\alpha$-periodic functions/distributions $m_{k}$ with bounded Fourier coefficients. Given $N \geq 2$, choose a sequence $m_{k}$ to be $N$-periodic, namely $m_{k+N}=m_{k}$ 
for all $k \in \mathbb{Z}$. Then

$$
\begin{aligned}
\sum_{k \in \mathbb{Z}} m_{k} T_{k / \beta} g & =\sum_{j=0}^{N-1} \sum_{l \in \mathbb{Z}} m_{j+l N} T_{(j+l N) \alpha / N} g \\
& =\sum_{j=0}^{N-1} m_{j} T_{j \alpha / N}\left(\sum_{l \in \mathbb{Z}} T_{l \alpha} g\right)=a \sum_{j=0}^{N-1} m_{j} .
\end{aligned}
$$

The latter sum vanishes with an appropriate choice of $m_{j}$, e.g., choose $m_{l N}=1$ and $m_{1+l N}=-1$ and $m_{j+l N}=0$ for $j=2, \ldots, N-1$ and $l \in \mathbb{Z}$. The corresponding sequence $\mathbf{c}$ is $c_{j+m N, l}=(-1)^{j} \delta_{l, 0}$ for $j=0,1$ and $l, m \in \mathbb{Z}$ and $c_{k l}=0$ otherwise. Of course, a similar statement can be formulated in dimension $d>1$.

If $g$ is the finite or infinite convolution product

$$
g=* \prod_{j=1}^{\infty} \chi_{\left[-a_{j} / 2, a_{j} / 2\right]}=\chi_{\left[-a_{1} / 2, a_{1} / 2\right]} * \chi_{\left[-a_{2} / 2, a_{2} / 2\right]} * \chi_{\left[-a_{3} / 2, a_{3} / 2\right]} * \ldots
$$

for $a_{j}>0$ and $\sum a_{j}<\infty$, then $\sum_{k \in \mathbb{Z}} g\left(x-a_{j} k\right)=b_{j} \neq 0$ for each $j$. Consequently, for any lattice of the form $\Lambda=\alpha \mathbb{Z} \times \beta \mathbb{Z}$ with $\alpha>0$ arbitrary and $\beta=N / a_{j}$, $j \in \mathbb{N}$ and $N=2,3, \ldots$, the Gabor system $\mathcal{G}(g, \Lambda)$ cannot be a frame for $L^{2}$. This example shows that the pattern of excluded frequency parameters $\beta$ can be made arbitrarily complicated.

A direct proof of the implication (xiii) $\Rightarrow(x i)$, (xii). We note this implication can be proved directly by using an observation of Y. Choi in a more general context. The sequence space $\ell^{1}\left(\Lambda^{\circ}\right)$ is a Banach algebra under twisted convolution $\downarrow$, and its dual is $\ell^{\infty}\left(\Lambda^{\circ}\right)$. Choi [5, Lemma 2] observed that if the (twisted) convolution operator $\mathbf{c} \rightarrow \mathbf{c} \downarrow \mathbf{s}$ is one-to-one on $\ell^{\infty}$, then the adjoint operator, which is again a convolution with a sequence $\mathbf{s}^{*}$ is surjective on $\ell^{1}\left(\Lambda^{\circ}\right)$. In the case of the Gramian, the twisted convolution is self-adjoint $\mathbf{s}=\mathbf{s}^{*}$, and so the injectivity of twisted convolution by $\mathbf{s}$ on $\ell^{\infty}$ implies its invertibility on $\ell^{1}$.

Structure of ker $D_{\Lambda^{\circ}} \ell^{\infty}$. If $D_{g, \Lambda^{\circ}}$ is non-trivial, then the kernel possesses special invariance properties that might be useful in order to disprove that a Gabor system is a frame.

Lemma 5.1. The subspace $\operatorname{ker} D_{g, \Lambda^{\circ}}$ is $w^{*}$-closed and an $\ell^{1}$-module under twisted convolution. Thus, if $\boldsymbol{a} \in \ell^{1}\left(\Lambda^{\circ}\right)$ and $\boldsymbol{c} \in \operatorname{ker} D_{g, \Lambda^{\circ}}$, then $\boldsymbol{a} \bigsqcup \boldsymbol{c} \in \operatorname{ker} D_{g, \Lambda^{\circ}}$.

Proof. Let $\mathbf{a} \in \ell^{1}\left(\Lambda^{\circ}\right)$ and $D_{g, \Lambda^{\circ}} \mathbf{c}=\sum_{\mu \in \Lambda^{\circ}} c_{\mu} \pi(\mu) g=0$ in $M^{\infty}$. Then as in (15) we find that

$$
\begin{aligned}
0 & =\pi(\mathbf{a}) \sum_{\mu \in \Lambda^{\circ}} c_{\mu} \pi(\mu) g \\
& =\pi(\mathbf{a}) \pi(\mathbf{c}) g=\pi(\mathbf{a} \downarrow \mathbf{c}) g \\
& =D_{g, \Lambda^{\circ}}(\mathbf{a} \downarrow \mathbf{c}),
\end{aligned}
$$

and thus $\mathbf{a} \measuredangle \mathbf{c} \in \operatorname{ker} D_{g, \Lambda^{\circ}}$. The $\mathrm{w}^{*}$-closedness is clear. 
The module property of ker $D_{g, \Lambda}$ suggests the definition of an index for a Gabor system $\mathcal{G}(g, \Lambda)$.

Definition 4. The index of the Gabor system $\mathcal{G}(g, \Lambda)$, denoted by ind $(g, \Lambda)$, is the smallest cardinality of a set of module generators of $\operatorname{ker} D_{g, \Lambda}$. We write ind $(g, \Lambda)=$ $N$, if there exist $N$ sequences $e_{j} \in \operatorname{ker} D_{g, \Lambda}$, such that every c $\in \operatorname{ker} D_{g, \Lambda}$ can be written as

$$
\mathbf{c}=\sum_{j=1}^{N} \mathbf{a}_{j} \downarrow e_{j}
$$

for some $\mathbf{a}_{j} \in \ell^{1}(\Lambda)$ and there is no set of smaller cardinality with this property.

With this definition we can recast the equivalence (i) $\Leftrightarrow$ (viii) as follows.

Theorem 5.2. The set $\mathcal{G}(g, \Lambda)$ is a frame for $L^{2}\left(\mathbb{R}^{d}\right)$ if and only if ind $\left(g, \Lambda^{\circ}\right)=0$.

At this point it is not clear what is the real significance of the index and what it says about rotation algebras and related objects. Here is what we know so far:

(a) If the time-frequency shifts $\{\pi(\lambda), \lambda \in \Lambda\}$ commute with each other, e.g. for the lattice $\Lambda=\mathbb{Z}^{2 d}$, then ker $D_{g, \Lambda}$ is a translation-invariant weak*-closed subspace of $\ell^{\infty}$. By a Tauberian theorem of Wiener, ker $D_{g, \Lambda}$ contains a sequence of the form $e_{\xi}(k)=e^{2 \pi i \xi \cdot k}, k \in \mathbb{Z}^{2 d}$. Furthermore, ind $\left(g, \mathbb{Z}^{2 d}\right)=\operatorname{card}\left\{\xi \in \mathbb{T}^{2 d}: e_{\xi} \in \operatorname{ker} D_{g, \Lambda}\right\}$.

(b) Since every lattice is of the form $\Lambda=A \mathbb{Z}^{2 d}$ for $A \in \mathrm{GL}(2 d, \mathbb{R})$, we may endow the set of lattices with the topology of $\mathrm{GL}(2 d, \mathbb{R})$. By a theorem of Feichtinger and Kaiblinger [17], the set of $g \in M^{1}$ and lattice $\Lambda$ for which $\mathcal{G}(g, \Lambda)$ is a frame is open in $M^{1} \times \operatorname{GL}(2 d, \mathbb{R})$. Thus by Theorem 5.2 the preimage $\operatorname{ind}^{-1}(\{0\})$ is open in $M^{1} \times \mathrm{GL}(2 d, \mathbb{R})$. This suggests that the index is a continuous function on $M^{1} \times \mathrm{GL}(2 d, \mathbb{R})$.

Other Types of Gabor Sets. The characterization of Theorem 3.1 can be extended to Gabor sets with several basis functions, so-called multi-window Gabor sets, and to vector-valued Gabor frames, so-called superframes. The set $\bigcup_{j=1}^{n} \mathcal{G}\left(g_{j}, \Lambda\right)$ is a frame, if for some constants $A, B>0$,

$$
A\|f\|_{2}^{2} \leq \sum_{\lambda \in \Lambda} \sum_{j=1}^{n}\left|\left\langle f, \pi(\lambda) g_{j}\right\rangle\right|^{2} \leq B\|f\|_{2}^{2} \quad \forall f \in L^{2}\left(\mathbb{R}^{d}\right) .
$$

The associated frame operator is $S=\sum_{j=1}^{n} S_{g_{j}, \Lambda}$. The same arguments as in the scalar case of Theorem 3.1 show that, for $g_{j} \in M^{1}, S$ is invertible on $L^{2}\left(\mathbb{R}^{d}\right)$ if and only if $S$ is invertible on $M^{p}$ for some/all $p, 1 \leq p \leq \infty$. The duality can be expressed as follows: let $\mathbf{g}=\left(g_{1}, g_{2}, \ldots, g_{n}\right)$ be the vector with component functions $g_{j}$ and $\pi(\lambda) \mathbf{g}=\left(\pi(\lambda) g_{1}, \ldots, \pi(\lambda) g_{n}\right)$ (action of $\pi$ is componentwise). Then $\bigcup_{j=1}^{n} \mathcal{G}\left(g_{j}, \Lambda\right)$ is a frame for $L^{2}\left(\mathbb{R}^{d}\right)$, if and only if $\mathcal{G}\left(\mathbf{g}, \Lambda^{\circ}\right):=\left\{\pi(\mu) \mathbf{g}: \mu \in \Lambda^{\circ}\right\}$ is a Riesz sequence in the space $L^{2}\left(\mathbb{R}^{d}, \mathbb{C}^{n}\right)$ of vector-valued functions. The Gramian of $\mathcal{G}\left(\mathbf{g}, \Lambda^{\circ}\right)$ has the entries $G_{\mu, \mu^{\prime}}=\left\langle\pi\left(\mu^{\prime}\right) \mathbf{g}, \pi(\mu) \mathbf{g}\right\rangle=\sum_{j=1}^{n}\left\langle\pi\left(\mu^{\prime}\right) g_{j}, \pi(\mu) g_{j}\right\rangle$, hence the same arguments as in Section 4.4 show that $G$ is invertible on $\ell^{2}\left(\Lambda^{\circ}\right)$ if and only if it is invertible on $\ell^{p}\left(\Lambda^{\circ}\right), 1 \leq p \leq \infty$. The conditions involving the coefficient and synthesis operator need a bit more care, because in this case they are different for $\Lambda$ and 
$\Lambda^{\circ}$. Specifically, $D_{\mathbf{g}, \Lambda}$ maps $\ell^{p}\left(\Lambda, \mathbb{C}^{n}\right)$ to $M^{p}$ by $D_{\mathbf{g}, \Lambda}\left(\mathbf{c}_{j}\right)=\sum_{\lambda \in \Lambda} \sum_{j=1}^{n} c_{\lambda, j} \pi(\lambda) g_{j}$, whereas $D_{\mathbf{g}, \Lambda^{\circ}}$ maps $\ell^{p}\left(\Lambda^{\circ}\right)$ to $M^{p}\left(\mathbb{R}^{d}, \mathbb{C}^{n}\right)$ by $D_{\mathbf{g}, \Lambda^{\circ}}(\mathbf{c})=\sum_{\mu \in \Lambda^{\circ}} c_{\mu} \pi(\mu) \mathbf{g}$. With these precautions all conditions of Theorem 3.1 can be formulated adequately and yield a characterization of "multi-window Gabor frames" without inequalities. We will return to this topic.

Finally, we mention that Theorem 3.1 carries over without change to characterize Gabor frames in arbitrary locally compact abelian groups.

\section{REFERENCES}

[1] W. Arveson. Discretized CCR algebras. J. Operator Theory, 26(2):225-239, 1991.

[2] M. J. Baastians. Gabor's expansion of a signal into Gaussian elementary signals. Proc. IEEE, 68:538-539, April 1980.

[3] R. Balan, P. G. Casazza, C. Heil, and Z. Landau. Density, overcompleteness, and localization of frames. II. Gabor systems. J. Fourier Anal. Appl., 12(3):309-344, 2006.

[4] V. Bargmann, P. Butera, L. Girardello, and J. R. Klauder. On the completeness of coherent states. Rep. Math. Phys., 2:221-228, 1971.

[5] Y. Choi. Injective convolution operators on $\ell^{\infty}(\gamma)$ are surjective. Preprint, 2006.

[6] O. Christensen. An introduction to frames and Riesz bases. Applied and Numerical Harmonic Analysis. Birkhäuser Boston Inc., Boston, MA, 2003.

[7] A. Connes. $C^{*}$ algèbres et géométrie différentielle. C. R. Acad. Sci. Paris Sér. A-B, 290(13):A599-A604, 1980.

[8] A. Connes. Noncommutative geometry. Academic Press Inc., San Diego, CA, 1994.

[9] I. Daubechies. Ten lectures on wavelets. Society for Industrial and Applied Mathematics (SIAM), Philadelphia, PA, 1992.

[10] I. Daubechies, H. J. Landau, and Z. Landau. Gabor time-frequency lattices and the WexlerRaz identity. J. Fourier Anal. Appl., 1(4):437-478, 1995.

[11] V. Del Prete. Estimates, decay properties, and computation of the dual function for Gabor frames. J. Fourier Anal. Appl., 5(6):545-562, 1999.

[12] H. G. Feichtinger. Un espace de Banach de distributions tempérées sur les groupes localement compacts abéliens. C. R. Acad. Sci. Paris Sér. A-B, 290(17):A791-A794, 1980.

[13] H. G. Feichtinger. On a new Segal algebra. Monatsh. Math., 92(4):269-289, 1981.

[14] H. G. Feichtinger. Atomic characterizations of modulation spaces through Gabor-type representations. In Proc. Conf. Constructive Function Theory, Edmonton, July 1986, pages $113-126,1989$.

[15] H. G. Feichtinger. Modulation spaces: looking back and ahead. Sampl. Theory Signal Image Process., 5(2):109-140, 2006.

[16] H. G. Feichtinger and K. Gröchenig. Gabor frames and time-frequency analysis of distributions. J. Functional Anal., 146(2):464-495, 1997.

[17] H. G. Feichtinger and N. Kaiblinger. Varying the time-frequency lattice of Gabor frames. Trans. Amer. Math. Soc., 356(5):2001-2023 (electronic), 2004.

[18] H. G. Feichtinger and W. Kozek. Quantization of TF lattice-invariant operators on elementary LCA groups. In Gabor analysis and algorithms, pages 233-266. Birkhäuser Boston, Boston, MA, 1998.

[19] H. G. Feichtinger and G. Zimmermann. A Banach space of test functions for Gabor analysis. In Gabor analysis and algorithms, pages 123-170. Birkhäuser Boston, Boston, MA, 1998.

[20] K. Gröchenig. Foundations of time-frequency analysis. Birkhäuser Boston Inc., Boston, MA, 2001.

[21] K. Gröchenig, A. J. E. M. Janssen, N. Kaiblinger, and G. E. Pfander. Note on B-splines, wavelet scaling functions, and Gabor frames. IEEE Trans. Inform. Theory, 49(12):3318-3320, 2003. 
[22] K. Gröchenig and M. Leinert. Wiener's lemma for twisted convolution and Gabor frames. J. Amer. Math. Soc., 17:1-18, 2004.

[23] K. Gröchenig and M. Leinert. Symmetry and inverse-closedness of matrix algebras and functional calculus for infinite matrices. Trans. Amer. Math. Soc., 358(6):2695-2711 (electronic), 2006.

[24] A. J. E. M. Janssen. Bargmann transform, Zak transform, and coherent states. J. Math. Phys., 23(5):720-731, 1982.

[25] A. J. E. M. Janssen. Duality and biorthogonality for Weyl-Heisenberg frames. J. Fourier Anal. Appl., 1(4):403-436, 1995.

[26] F. Luef. On spectral invariance of non-commutative tori. In Operator Theory, Operator Algebras, and Applications, volume 414, pages 131-146. American Mathematical Society, 2006.

[27] J. v. Neumann. Mathematische Grundlagen der Quantenmechanik. Springer, Berlin, 1932. English translation: "Mathematical foundations of quantum mechanics," Princeton Univ. Press, 1955.

[28] M. A. Rieffel. Projective modules over higher-dimensional noncommutative tori. Canad. J. Math., 40(2):257-338, 1988.

[29] A. Ron and Z. Shen. Weyl-Heisenberg frames and Riesz bases in $L_{2}\left(\mathbb{R}^{d}\right)$. Duke Math. J., 89(2):237-282, 1997.

Faculty of Mathematics, University of Vienna, Nordbergstrasse 15, A-1090 ViENNA, Austria

E-mail address: karlheinz.groechenig@univie.ac.at 